\title{
Talbot effect with rough reflection gratings
}

\author{
Francisco Jose Torcal-Milla, ${ }^{*}$ Luis Miguel Sanchez-Brea, and Eusebio Bernabeu \\ Applied Optics Complutense Group, Department of Optics, Universidad Complutense de Madrid, Facultad de Ciencias \\ Físicas, Ciudad Universitaria s.n., 28040, Madrid, Spain \\ ${ }^{*}$ Corresponding author: ftorcalmilla@fis.ucm.es
}

Received 30 October 2006; revised 12 February 2007; accepted 12 February 2007; posted 15 February 2007 (Doc. ID 76578); published 31 May 2007

\begin{abstract}
The Talbot effect is analyzed when steel tape gratings are used. These gratings are made on a steel substrate, and, because of the manufacture process, both levels of the grating are rough with different roughness parameters. A theoretical analysis based on Fresnel regime, which considers the statistical properties of roughness, is developed. Analytical formulas that show a decreasing exponential dependence on the intensity in terms of the distance between the grating and the observation plane are obtained, and an experimental verification is also performed. (C) 2007 Optical Society of America

OCIS codes: $\quad 050.0050,030.5770,050.2770,290.5880$.
\end{abstract}

\section{Introduction}

When a grating is illuminated with a monochromatic light, self-images of the grating are observed in the Fresnel regime at distances given by $z_{T}=n p^{2} / \lambda$, where $p$ is the period of the grating, $\lambda$ is the wavelength of the incident light, and $n$ is a natural number [1-3]. This is known as the Talbot effect. The interest in the Talbot effect is not only theoretical. It can also be found in a multitude of applications such as spectroscopy, optical metrology, moiré interferometry, laser array illumination, and phase locking of the laser array [4-7]. For this reason, several works on the subject have been performed recently analyzing the Talbot effect for the oblique angle of light propagation [8], polychromatic light [9], partial coherent light illumination [10], when the grating presents different kinds of flaws [11], and when polarized light is used [12].

In most applications, glass gratings are used because of its good behavior, with periods ranging from hundred of micrometers to lower than $1 \mu \mathrm{m}$. However, there exist applications in which very long displacements need to be measured $(>3 \mathrm{~m})$. In these cases, glass gratings are not available since they are difficult to manufacture and handle. As a consequence, steel substrates are used. Gratings on a steel substrate experimentally present a worse behavior

0003-6935/07/183668-06\$15.00/0

(C) 2007 Optical Society of America owing to their fabrication process. The steel tape where the grating is engraved is not perfectly smooth, but it presents a certain roughness. Also, the technique to engrave the slits produces much rougher surfaces.

The period of gratings on a steel substrate is larger than in chrome on glass gratings (approximately $100 \mu \mathrm{m})$. Owing to this long period, the Talbot effect is not observed [Fig. 1(a)], and steel reflection gratings are normally studied under a geometrical optics approach. However, today we can find steel tape gratings with periods below $40 \mu \mathrm{m}$, and, as a consequence, diffractive effects are more important. Talbot distances present a quadratic dependence with the period of the grating. As a result, the Talbot effect becomes a matter for the mechanical tolerances of the devices.

In this work, we have analyzed the effect of roughness in the self-imaging process of steel reflection gratings. We have modeled the grating as an amplitude binary grating with roughness in only one of the two levels of the grating. This assumption is motivated by the experimental evidence that when the grating is imaged using a CCD camera and a microscope objective, only one level of the grating presents a significant intensity [Fig. 1(b)]. The reason for this effect is that one of the grating levels is very rough, and it scatters light into all directions. As expected, the Talbot effect appears. Owing to the random topography of the surfaces, statistical techniques are 


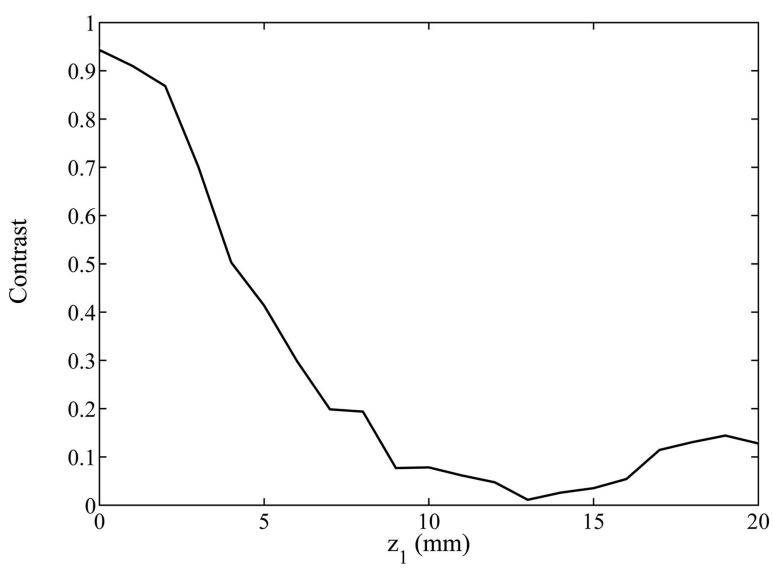

(a)

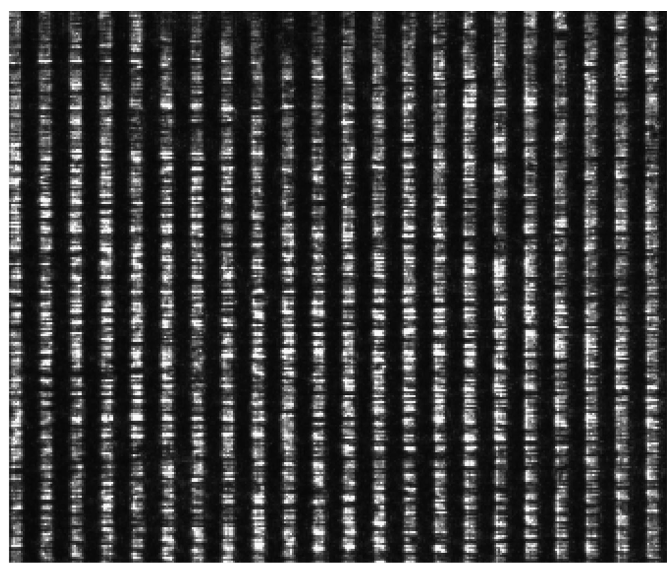

(b)

Fig. 1. (a) Experimental contrast of fringes for a $100 \mu \mathrm{m}$ steel grating in terms of the distance between the grating and the observation plane. (b) Image of the grating using the experimental setup used in Section 3. The levels of the grating with a shorter correlation length present a low-intensity since light is scattered in all directions.

required to determine the intensity of the selfimages. We have theoretically found that the contrast of the self-images decreases as we separate from the grating. Experimental results are obtained that corroborate the dependence of the contrast of self-images with the distance. A comparison of the experimental results with the theoretical model shows that a decrease of the contrast of the self-images is due to the level of the grating whose roughness presents a longer correlation length.

We have also found experimentally that the fringes obtained become more sinusoidal as the number of self-images increases. An explanation of this effect, based on the equations obtained, is also performed. This effect may be important for applications where the arctangent algorithm is used to determine parameters from the fringes.

\section{Theoretical Analysis}

Let us consider a monochromatic plane wave with amplitude $A_{0}$ propagating over the $z$ coordinate, perpendicular to the grating (Fig. 2). The grating is

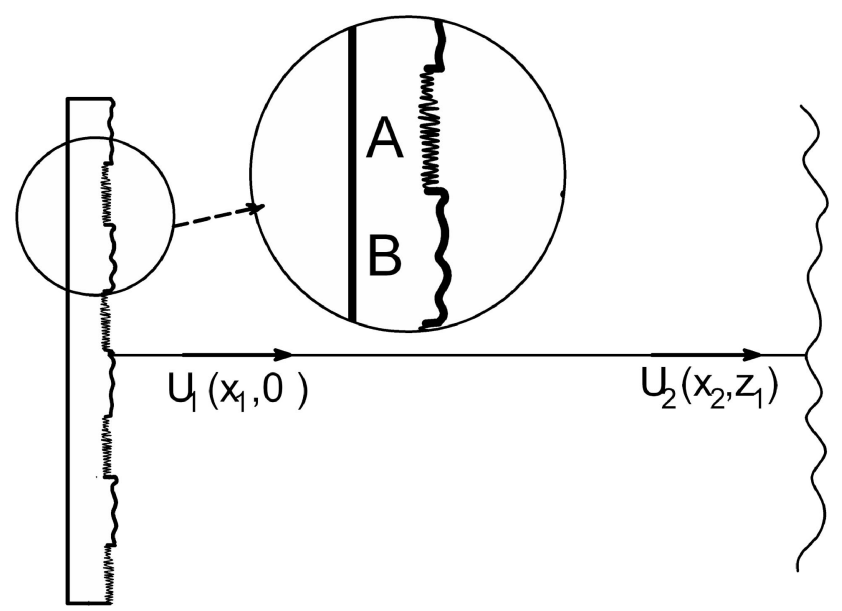

Fig. 2. Scheme showing the notation used. placed at $z=0$. We will consider that the period of the grating $p$ is much larger than the wavelength, thus a scalar treatment is possible. In addition we will assume that the grating is binary, characterized by two levels with different roughness (levels $\mathrm{A}$ and $\mathrm{B}$, as defined in Fig. 2). Level A presents roughness with a correlation length $T_{A}$ shorter than the period of the grating, $T_{A}<p$. Level $\mathrm{B}$ also presents roughness, with its correlation length $T$ much larger than the period of the grating $T>p$. Because of its high roughness, the light that reaches level $\mathrm{A}$ is scattered into all directions incoherently, and only a small fraction reaches an observation plane at a certain distance from the grating. This grating can be mathematically modeled as a multiplication of two factors $T\left(x_{1}\right)$ $=g\left(x_{1}\right) t\left(x_{1}\right)$. The first factor accounts for an amplitude grating, defined as $g\left(x_{1}\right)=\sum_{n} a_{n} \exp \left(\right.$ inq $\left.x_{1}\right)$ where $a_{n}$ are the Fourier coefficients of the grating, $q=2 \pi / p$, and $x_{1}$ is the transversal coordinate at the grating plane. The second factor $t\left(x_{1}\right)$ represents the reflectance of level B. The topography $\zeta\left(x_{1}\right)$ of level B is random, whose average height is null, $\left\langle\zeta\left(x_{1}\right)\right\rangle=0$. To describe such a roughness we will assume a normal distribution in heights $w(z)=\exp \left(-z^{2} / 2 \sigma^{2}\right) / \sqrt{2 \pi} \sigma$ where $z=\zeta\left(x_{1}\right)$, and $\sigma$ is the standard deviation [13]. Then the reflectance results $t\left(x_{1}\right)=\exp \left[i 2 k \zeta\left(x_{1}\right)\right]$, where $k=2 \pi / \lambda$. The characteristic function is then $\langle t(x)\rangle=\exp [-g / 2]$, where $g=(4 \pi \sigma / \lambda)^{2}$. The correlation properties of roughness are defined below.

The amplitude after the grating results in

$$
U\left(x_{1}\right)=A_{0} t\left(x_{1}\right) \sum_{n} a_{n} \exp \left(\text { iqn }_{1}\right) .
$$

Assuming that the Fresnel approach is valid for the propagation, the field at $z_{1}$ results in

$$
\begin{aligned}
U_{2}\left(x_{2}\right)= & A_{0} \sum_{n} a_{n} \int t\left(x_{1}\right) \exp \left(i q n x_{1}\right) \\
& \times \exp \left[-\left(i k / 2 z_{1}\right)\left(x_{2}-x_{1}\right)^{2}\right] d x_{1},
\end{aligned}
$$




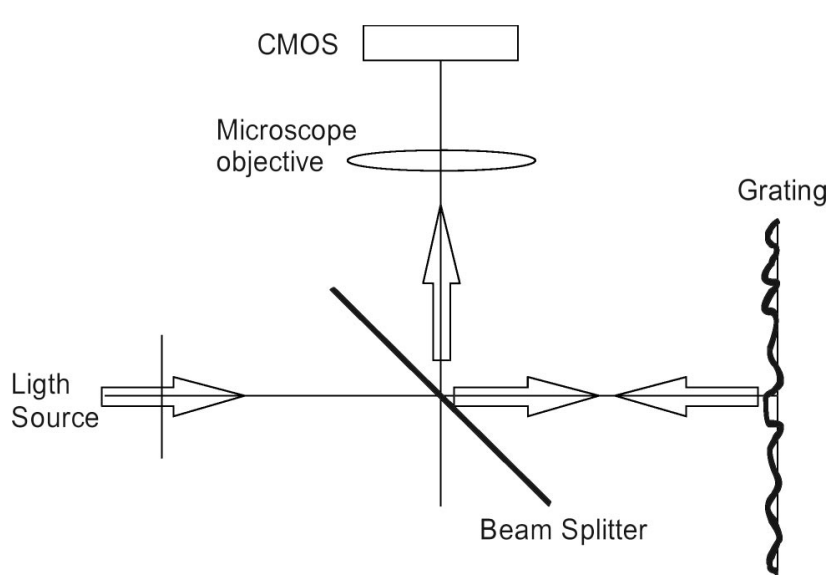

Fig. 3. Experimental setup for reflection configuration.

where $x_{2}$ is the transversal coordinate at the observation plane. Then the average intensity is obtained as $\left\langle I\left(x_{2}\right)\right\rangle=\left\langle U_{2}\left(x_{2}\right) U_{2}^{*}\left(x_{2}\right)\right\rangle$, resulting in

$$
\begin{aligned}
\left\langle I_{2}\left(z_{1}\right)\right\rangle= & \left|A_{0}\right|^{2} \sum_{n} \sum_{n^{\prime}} a_{n} a_{n^{\prime}}{ }^{*} \iint \exp \left(i q n x_{1}\right) \exp \left(-i q n^{\prime} x_{1}{ }^{\prime}\right) \\
& \times\left\langle t\left(x_{1}\right) t^{*}\left(x_{1}{ }^{\prime}\right)\right\rangle \exp \left[\left(i k / 2 z_{1}\right)\left(x_{2}-x_{1}{ }^{\prime}\right)^{2}\right] \\
& \times \exp \left[-\left(i k / 2 z_{1}\right)\left(x_{2}-x_{1}\right)^{2}\right] d x_{1} d x_{1}{ }^{\prime},
\end{aligned}
$$

where we have considered that the only stochastic factor in this equation is $\left\langle t\left(x_{1}\right) t^{*}\left(x_{1}{ }^{\prime}\right)\right\rangle$. An exact description of this topography is not known. However, the autocorrelation function of the surface is measurable.

This autocorrelation function is usually modeled as a Gaussian function, decreasing the exponential function or other functions that fit the experimental results and allow an analytical integration of the equations involved [13-16]. In this work we have used the decreasing exponential model

$$
\left\langle t\left(x_{1}\right) t^{*}\left(x_{1}{ }^{\prime}\right)\right\rangle=\exp \left[-\frac{\left|x_{1}-x_{1}{ }^{\prime}\right|}{T_{0}}\right]
$$

where we have assumed a strong diffuser with exponential distribution of heights and also an exponential correlation function, where $T_{0}$ is the correlation length of the field after the grating. Using this high roughness approach, $g \gg 1$, we can relate the field correlation length $T_{0}$ with the roughness parameters, resulting in [16]

$$
T_{0}=\frac{\lambda T}{4 \pi \sigma} .
$$

Substituting Eq. (4) into Eq. (3), and performing the integrations, the average intensity at the observation plane is

$$
\begin{aligned}
\left\langle I_{2}\left(z_{1}\right)\right\rangle= & \left|A_{0}\right|^{2} \sum_{n} \sum_{n^{\prime}} a_{n} a_{n^{\prime}}{ }^{*} \exp \left[i q x_{2}\left(n^{\prime}-n\right)\right] \\
& \times \exp \left[i \frac{q^{2}}{2 k}\left(n^{\prime 2}-n^{2}\right) z_{1}\right] \exp \left[-\frac{\lambda z_{1}}{p T_{0}}\left|n-n^{\prime}\right|\right] .
\end{aligned}
$$

(a)

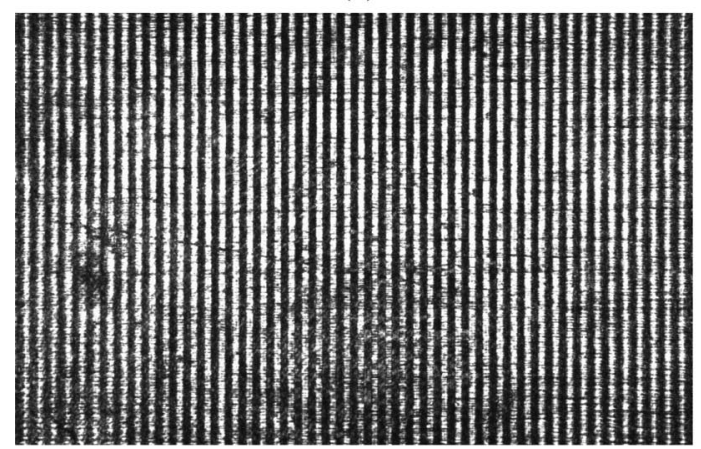

(c)

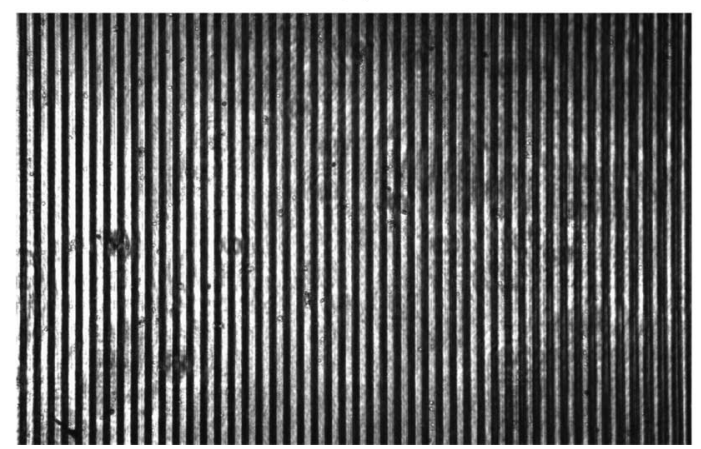

(b)

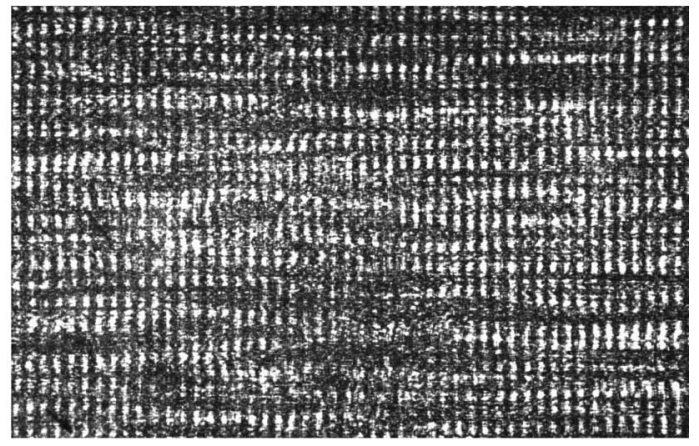

(d)

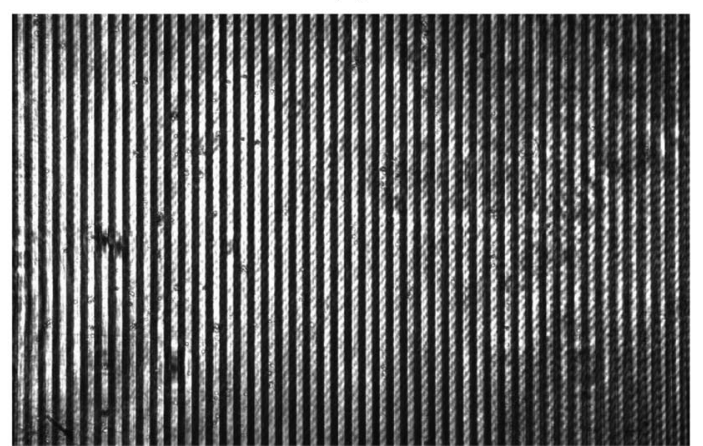

Fig. 4. Self-images obtained for steel (a), (b) and glass (c), (d) gratings. (a) and (c) are the first self-images of the gratings obtained with the microscope, and (b) and (d) are the third self-images. 
The first exponential factor is related to the period of the grating, the second factor accounts for the Talbot effect, and the third exponential factor accounts for the effect of roughness on the self-images intensity. The roughness at the grating makes the intensity decrease exponentially as the distance $z_{1}$ increases. The distance at which the intensity decays $1 / e$ for the first harmonic results $W=p T_{0} / \lambda$.

There exist experimental cases where the autocorrelation function of the surface cannot be fitted to an exponential, but to a Gaussian function $\left\langle t\left(x_{1}\right) t^{*}\left(x_{1}{ }^{\prime}\right)\right\rangle$ $=\exp \left[-\left(x_{1}-x_{1}{ }^{\prime}\right)^{2} / T_{0}{ }^{2}\right]$. Then Eq. (3) can also be analytically solved, resulting in

$$
\begin{aligned}
\left\langle I_{2}\right\rangle= & \left|A_{0}\right|^{2} \sum_{n} \sum_{n^{\prime}} a_{n} a_{n^{*}}{ }^{*} \exp \left[i q x_{2}\left(n^{\prime}-n\right)\right] \\
& \times \exp \left[i \frac{q^{2}}{2 k}\left(n^{\prime 2}-n^{2}\right) z_{1}\right] \exp \left[-\left(\left(n-n^{\prime}\right) \frac{\lambda z_{1}}{p T_{0}}\right)^{2}\right] .
\end{aligned}
$$

The structure of this equation is equivalent to Eq. (6), substituting the exponential function for a Gaussian function.

\section{Experimental Approach}

The experimental setup is shown in Fig. 3. A monochromatic collimated laser beam of wavelength

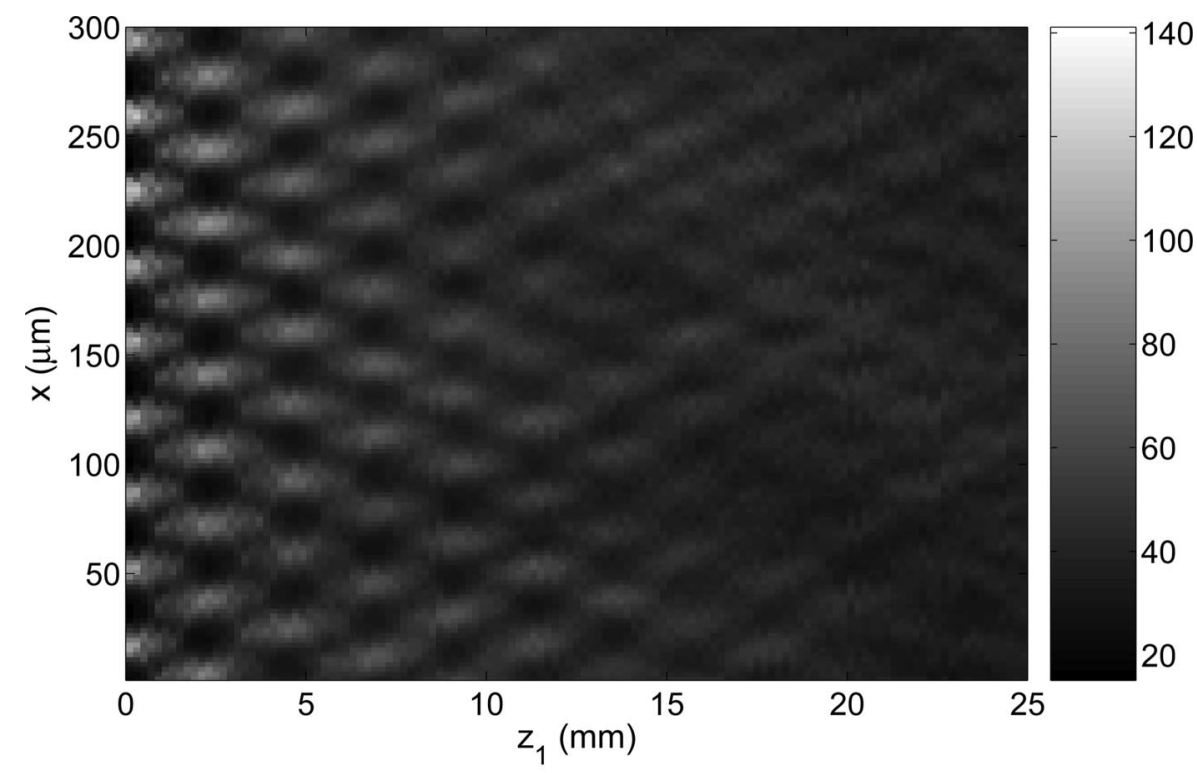

(a)

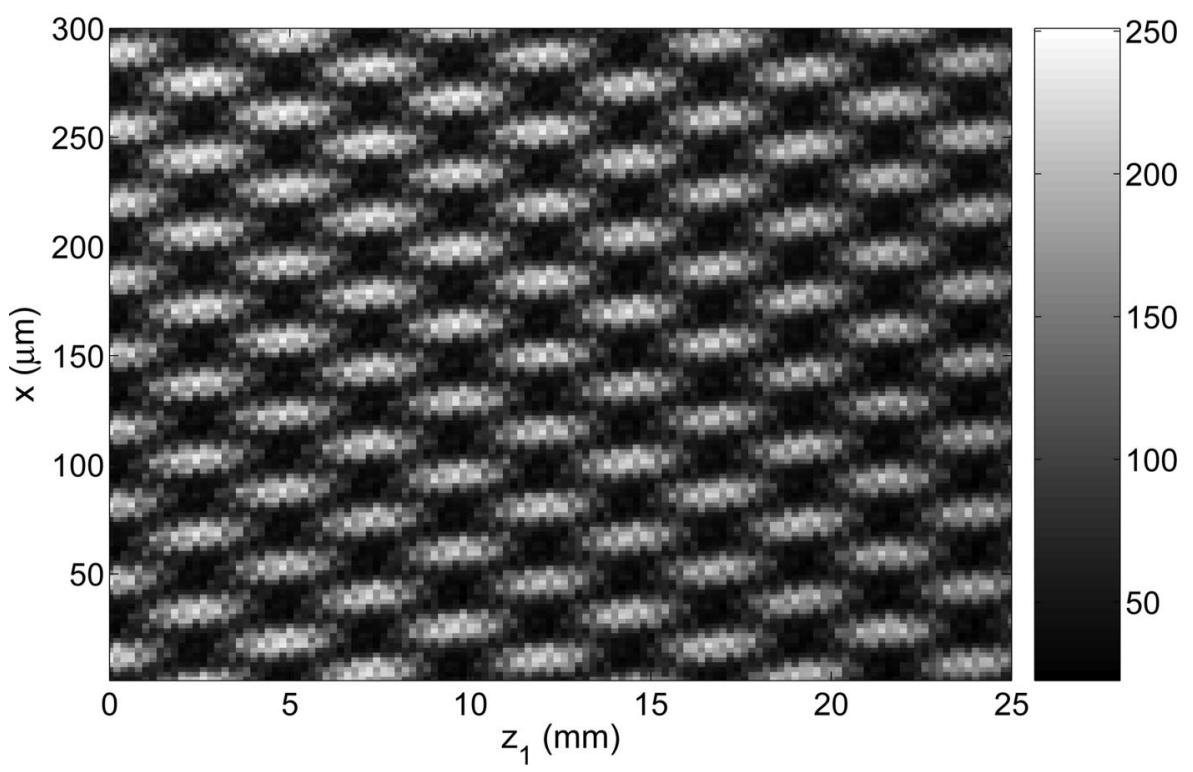

(b)

Fig. 5. Fringes in terms of the distance between the grating and the observation plane for (a) the steel rough grating and (b) the chrome on glass grating. 
$675 \mathrm{~nm}$ (MC6707O model by Monocrom, Cataluña, Spain) impinges on a steel tape grating (period 40 $\mu \mathrm{m}$ ) with normal incidence with respect to its surface. The steel tape grating is placed on a motorized linear stage along the $z$ direction. The diffracted light crosses the beam splitter, and it is collected by a complementary metal-oxide semiconductor (CMOS) camera (UI-1220-M model by uEye, Obersulm, Germany, pixel size $6 \times 6 \mu \mathrm{m}$ ) with a $10 \times$ objective microscope. With this setup, we can measure the intensity of all the self-images. The intensity distribution at the observation plane is not very uniform owing to speckle produced by the rough surface. As an example, in Fig. 4 we show the first and third selfimages.

We have also determined the evolution of the Talbot planes in terms of the $z_{1}$ distance between the grating and the observation plane. To obtain the intensity of the self-images we have performed a vertical averaging. In Fig. 5(a) we can see the fringes in terms of the distance $z_{1}$. The intensity of the selfimages decreases for the steel grating in terms of the distance $z_{1}$. For comparison, we have analyzed the intensity of the self-images obtained with a chrome on glass grating (with a period of $40 \mu \mathrm{m}$ ) in Fig. 5(b). In this case, there is not an observable decreasing of the self-images with the distance.

We have also determined the contrast of the fringes for different distances $z$ between the grating and the observation plane. We have used the standard definition of contrast, given by $C=\left(I_{\max }-I_{\min }\right) /\left(I_{\max }\right.$ $+I_{\min }$ ) where $I_{\max }$ and $I_{\min }$ are the maximum and minimum intensities for each observation plane. In Fig. 6, the contrast for the steel reflection grating is shown. An exponential diminution of the contrast with the distance can be clearly observed.

We have also measured the topography of the steel grating with a confocal microscope (PL $\mu$ by Sensofar, Barcelona, Spain) in Fig. 7. The correlation length for the rough part (level A) of the grating

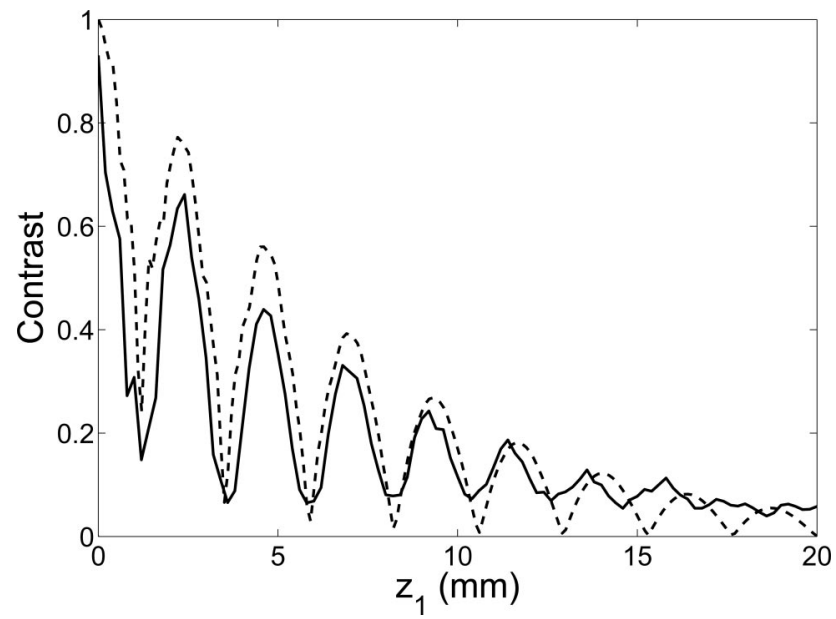

Fig. 6. Experimental contrast in terms of the distance for the steel rough surface (solid) and theoretical contrast obtained with Eq. (6) (dashed). In the numerical approach, we have truncated the infinite series to the interval $n=n^{\prime}=-5, \ldots, 5$.

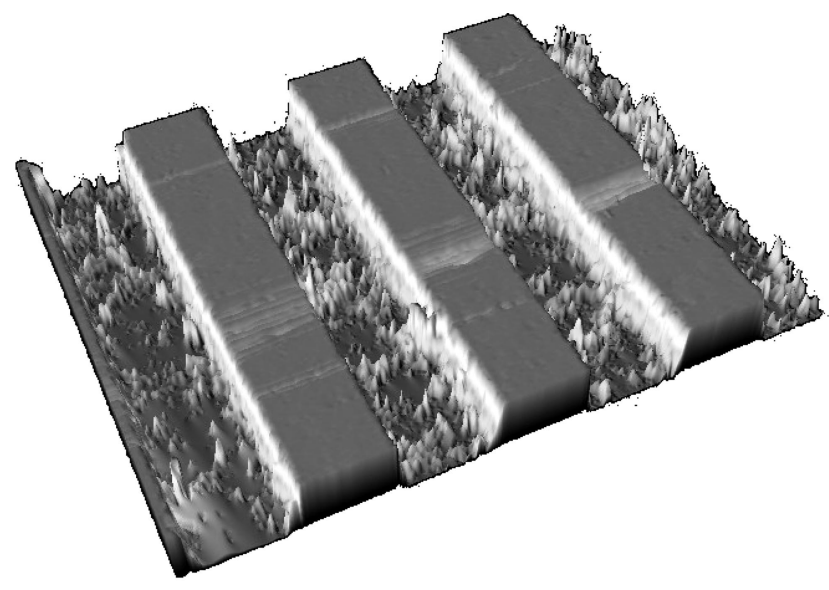

(a)

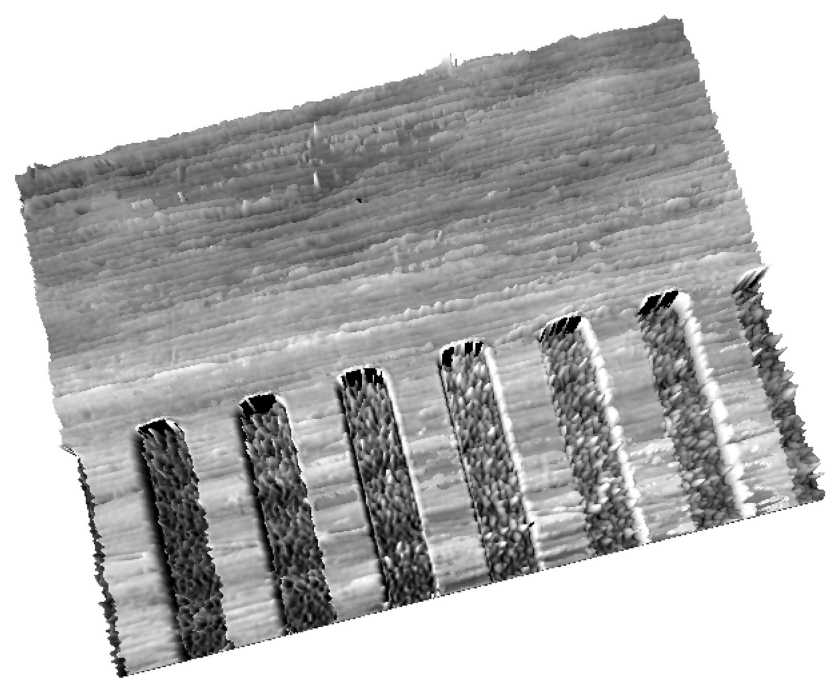

(b)

Fig. 7. (a) Image obtained with confocal microscopy of the steel rough grating. (b) Same steel grating showing that the statistical parameters of level B are the same as the zone of the steel tape where the grating is not engraved.

was $T=1.42 \mu \mathrm{m}$, and standard deviation $\sigma=$ $0.2 \mu \mathrm{m}$. As a consequence $T / \sigma=7.1$. The level $\mathrm{B}$ roughness parameters have also been measured. Since the correlation length for this part is much larger than the period of the grating, we have determined the roughness parameters using a zone of the steel tape where the grating is not engraved [Figure 7(b)]. Due to the fabrication process, it has the same statistical properties. We have found that $T$ $=276 \mu \mathrm{m}$ and $\sigma=0.21 \mu \mathrm{m}$, and then $T / \sigma=1314$.

Using these data, the decay of contrast given in Fig. 5(a) is compared to that obtained using the theoretical approach [Eq. (6)]. Since the width is $W=$ $p T_{0} / \lambda$, and considering Eq. (5), it results $W=$ $p T / 4 \pi \sigma$, which is independent on the wavelength. Introducing the experimental parameters we find that $W=4.2 \mathrm{~mm}$, which is close to the experimental value obtained using Fig. 6. In this figure we have also included the theoretical contrast obtained using these parameters. 


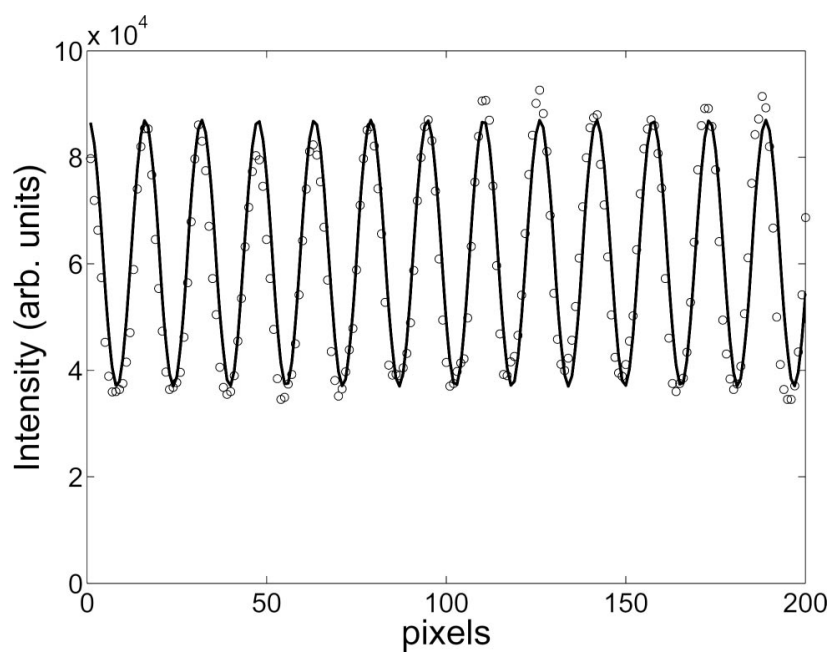

Fig. 8. Experimental fringes obtained for the third self-image of the steel reflection grating and fit to a sinusoidal function.

\section{A. Shape of Fringes}

We have also found that the fringes produced by the steel grating can be adjusted to a sinusoidal function (Fig. 8). This is quite interesting in applications that use the fringes in the arctangent technique. This effect can be explained in terms of Eq. (6). Each term of the summation presents a different width of the exponential factor given by $W_{n, n^{\prime}}=k T_{0} / q\left|n-n^{\prime}\right|$. As a consequence, when $\left|n-n^{\prime}\right|$ increases, then the width of the exponential for this factor decreases, and eventually only the terms with a difference $\left|n-n^{\prime}\right|=1$ will survive for long values of $z_{1}$. Since all the terms with $\left|n-n^{\prime}\right|=1$ present the same period, as it is shown by the $\exp \left[i q x_{2}\left(n^{\prime}-n\right)\right]$ term, then the fringes will be sinusoidal.

\section{Conclusions}

In this work we have theoretically analyzed the Talbot effect when the grating is rough. We have shown that the roughness of the surface makes the average intensity at the observation plane decrease exponentially with the distance. An experimental verification of this effect is performed, which is in agreement with the theoretical expressions. In addition, we have experimentally found that the fringes of the self-images become more sinusoidal for long distances. This effect can be explained in terms of the theoretical approach.
This work has been supported by the DPI200502860 project of the Ministerio de Educación y Ciencia of Spain and the "Tecnologías en ecología, alta precisión y productividad, multifuncionalidad, y tecnologías de la información y comunicaciones en Máquina Herramienta" CENIT project of the Ministerio de Industria, turismo y comercio. Sanchez-Brea is currently contracted by the Universidad Complutense de Madrid under the "Ramón y Cajal" research program of the Ministerio de Educación y Ciencia of Spain.

\section{References}

1. W. H. F. Talbot, "Facts relating to optical science," Philos. Mag. 9, 401-407 (1836).

2. K. Patorski, "The self-imaging phenomenon and its applications," Prog. Opt. 27, 1-108 (1989).

3. E. Keren and O. Kafri, "Diffraction effects in moiré deflectometry,” J. Opt. Soc. Am. A 2, 111-120 (1985)

4. A. W. Lohmann and D. E. Silva, "An interferometer based on the Talbot effect," Opt. Commun. 2, 413-415 (1971).

5. G. Schirripa Spagnolo, D. Ambrosini, and D. Paoletti, "Displacement measurement using the Talbot effect with a Ronchi grating," J. Opt. A, Pure Appl. Opt. 4, S376-S380 (2002).

6. B. F. Oreb and R. G. Dorsch, "Profilometry by phase-shifted Talbot images," Appl. Opt. 33, 7955-7962 (1994).

7. S. Wei, S. Wu, I. Kao, and F. P. Chiang, "Measurement of wafer surface using shadow moiré technique with Talbot effect," Trans. ASME J. Electron. Packag. 120, 166-170 (1998).

8. M. Testorf, J. Jahns, N. A. Khilo, and A. M. Goncharenko, "Talbot effect for oblique angle of light propagation," Opt. Commun. 129, 167-172 (1996).

9. N. Guérineau, B. Harchaoui, and J. Primot, "Talbot experiment re-examined: demonstration of an achromatic and continuous self-imaging regime," Opt. Commun. 180, 199-203 (2000).

10. S. Teng, L. Liu, J. Zu, Z. Luan, and De'an, "Uniform theory of the Talbot effect with partially coherent light illumination," J. Opt. Soc. Am. A 20, 1747-1754 (2003).

11. Y. Lu, C. Zhou, and H. Luo, "Talbot effect of a grating with different kinds of flaws," J. Opt. Soc. Am. A 22, 2662-2667 (2005).

12. Y. Lu, C. Zhou, S. Wang, and B. Wang, "Polarizationdependent Talbot effect," J. Opt. Soc. Am. A 23, 2154-2160 (2006).

13. P. Beckmann and A. Spizzichino, The Scattering of Electromagnetic Waves from Rough Surfaces (Artech House, 1987).

14. J. C. Dainty, Laser Speckle and Related Phenomena (SpringerVerlag, 1984).

15. J. A. Ogilvy, Theory of Wave Scattering from Random Rough Surfaces (Institute of Physics, 1991).

16. F. Perez-Quintián, A. Lutenberg, and M. A. Rebollo, "Linear displacement measurement with a grating and speckle pattern illumination," Appl. Opt. 45, 4821-4825 (2006). 